\title{
Status of a flavor-maximal nonminimal universal extra dimension model
}

\section{Sayan Dasgupta*}

Centre for Theoretical Studies, Indian Institute of Technology, Kaharagpur

E-mail: sayandg05@gmail.com

In this paper we consider an $S^{1} / \mathbb{Z}_{2}$ compactified flat extra dimensional scenario where all the standard model states can access the bulk and have generalised brane localised kinetic terms. The flavour structure of brane kinetic terms for the standard model fermions are dictated by stringent flavour bounds on the first two generations implying an $U(2)_{Q_{L}} \otimes U(2)_{u_{R}} \otimes U(2)_{d_{R}}$ flavour symmetry. We consider the constraints on such a scenario arising from dark matter relic density and direct detection measurements, precision electroweak data, Higgs physics and LHC dilepton searches. We discuss the possibility of such a scenario providing an explanation of the recently measured anomaly in $R_{K^{(*)}}$ within the allowed region of the parameter space.

ICHEP2018 SEOUL, XXXIX INTERNATIONAL CONFERENCE ON HIGH ENERGY PHYSICS

5-11 July 2018

Seoul, South Korea

${ }^{*}$ Speaker. 


\section{Set-up}

We will consider that the extra spatial dimension is compactified on an $S^{1} / \mathbb{Z}_{2}$ manifold and all standard model states can access the bulk. The end points are locations of the 3-branes with symmetric brane localized terms as detailed in [1]. Schematically the fivedimensional action for the SM quarks is given by,

\begin{tabular}{|c|c||c|c|}
\hline$\left(\begin{array}{c}v_{e} \\
e\end{array}\right)_{L}, e_{R}$ & $r_{f_{1}}$ & $\left(\begin{array}{l}u \\
d\end{array}\right)_{L}, u_{R}, d_{R}$ & $r_{f_{1}}$ \\
\hline$\left(\begin{array}{c}v_{\mu} \\
\mu\end{array}\right)_{L}, \mu_{R}$ & $r_{f_{2}}$ & $\left(\begin{array}{l}c \\
s\end{array}\right)_{L}, c_{R}, s_{R}$ & $r_{f_{1}}$ \\
\hline$\left(\begin{array}{c}v_{\tau} \\
\tau\end{array}\right)_{L}, \tau_{R}$ & $r_{f_{3}}$ & $\left(\begin{array}{l}t \\
b\end{array}\right)_{L}, t_{R}, b_{R}$ & $r_{f_{3}}$ \\
\hline$W_{\mu}, B_{\mu}, G_{\mu}, H$ & $r_{g}$ & Yukawa & $r_{y}$ \\
\hline
\end{tabular}

Figure 1: Choice of BLT parameters.

$$
\mathscr{S}_{\text {quark }}=\sum_{Q, U, d, L, v} \int d^{4} x \int_{0}^{\pi R} d y\left[i \bar{\psi} \Gamma^{M} \mathscr{D}_{M} \psi+r_{f}\{\delta(y)+\delta(y-\pi R)\} i \bar{\psi} \gamma^{\mu} \mathscr{D}_{\mu} P_{L / R} \psi\right],
$$

The BLKT set-up used in the phenomenological studies in this paper has been summarised in Figure 1.

\section{Results}

The constraints have been imposed in the following order and are depicted in Figure 2 with different colours: Flavor constraints (namely $R_{K^{(*)}}$ ) [2], Dark matter relic density and direct detection constraints [3], Constraints from Higgs data measurements and precision measurements [4] [5], and Direct LHC constraints from the dilepton channel [6]. Only three benchmark points out of 2 million survive all the constraints (indicating a survival fraction of $2 \times 10^{-6}$ ). This shows that the model is highly constrained from various experimental observations.

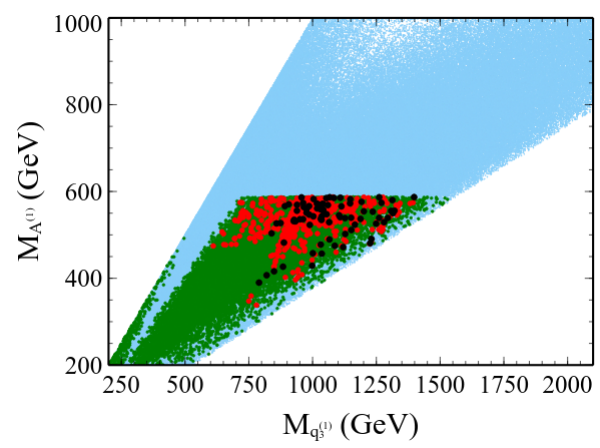

\section{References}

\begin{tabular}{|c|c|c|c|c|c|c|c|c|c|}
\hline & $R_{g}$ & $R_{f_{1}}$ & $R_{f_{2}}$ & $R_{f_{3}}$ & $\begin{array}{c}M_{A^{(1)}} \\
(\mathrm{GeV})\end{array}$ & $\begin{array}{c}M_{q_{3}^{(1)}} \\
(\mathrm{GeV})\end{array}$ & $R_{K}$ & $R_{K^{*}} / R_{K}$ & $\Omega h^{2}$ \\
\hline \hline BP-I & 5.098 & 3.922 & 2.966 & 0.2385 & 540.8 & 1108 & 0.924 & 1.034 & 0.101 \\
\hline BP-II & 6.404 & 4.997 & 4.087 & 0.0281 & 539.1 & 1295 & 0.9358 & 1.028 & 0.101 \\
\hline BP-III & 7.788 & 6.102 & 4.746 & 0.3496 & 525.8 & 1249 & 0.9279 & 1.032 & 0.0958 \\
\hline
\end{tabular}

Figure 2: Above: Allowed parameter points which are within $2 \sigma$ of $R_{K^{(*)}}$ and are allowed by other constraints are presented in the parameter space of $M_{A^{(1)}}-M_{q_{3}^{(1)}}$. Below: Benchmark points that survive all constraints.

[1] S.Dasgupta, U.K.Dey, T.Jha and T.S.Ray, Phys.Rev. D 98 (2018) no.5, 055006 [arXiv:1801.09722].

[2] LHCb Collaboration, R. Aaij et al., Phys. Rev. Lett. 113 (2014) 151601, JHEP 08 (2017) 055.

[3] LUX Collaboration, D. S. Akerib et al., Phys. Rev. Lett. 118 (2017), no. 25 251302, XENON Collaboration, E. Aprile et al., arXiv:1805.12562.

[4] J. Ellis and T. You, JHEP 06 (2013) 103, [arXiv:1303.3879].

[5] Gfitter Group Collaboration, Eur. Phys. J. C74 (2014) 3046, [arXiv:1407.3792].

[6] ATLAS Collaboration, Tech. Rep. ATLAS-CONF-2016-045, CERN, Geneva, Aug, 2016, CMS Collaboration, Tech. Rep. CMS-PAS-EXO-16-031, CERN, Geneva, 2016. 